The Astrophysical Journal, 390:L89-L91, 1992 May 10

(C) 1992. The American Astronomical Society. All rights reserved. Printed in U.S.A.

\title{
THE DISCOVERY OF A NEW DAV STAR USING IUE TEMPERATURE DETERMINATION
}

\author{
A. KanAan, ${ }^{1,2,3}$ S. O. KePleR ${ }^{1}$, AND O. GiOVANNINI ${ }^{1,2}$ \\ Instituto de Física, Universidade Federal do Rio Grande do Sul, 91500-Porto Alegre, RS-Brazil \\ AND \\ M. DIAZ ${ }^{1,4}$ \\ Instituto Astronômico e Geofísico, Universidade de São Paulo, 04301-São Paulo, SP-Brazil \\ Received 1991 December 27; accepted 1992 February 19
}

\begin{abstract}
We report the discovery of a new ZZ Ceti star observed on the assumption that most DA stars with temperature near $11,000 \mathrm{~K}$ are pulsators and on a temperature determination using IUE data. Even though BPM 37093 had already been claimed as a nonvariable, it pulsates with a semi-amplitude of 4 mmag and a period of $\sim 600 \mathrm{~s}$, being the smallest amplitude ZZ Ceti star known to date.

Subject headings: stars: oscillations — stars: variable: other — white dwarfs
\end{abstract}

\section{INTRODUCTION}

The first ZZ Ceti star was discovered by Landolt in 1968 , who serendipitously identified the star HL Tau 76 as variable. The ZZ Ceti stars are the variable white dwarfs with pure hydrogen atmospheres; they are also called DAVs (Sion et al. 1983). Since Robinson (1976) showed that the ZZ Ceti stars are in a region of the H-R diagram which is an extension of the Cepheid instability strip, many DAs have been observed to test whether every DA inside the instability pulsates or not. McGraw (1979), Fontaine et al. (1982), Greenstein (1982), and Fontaine et al. (1985), show the existence of a narrow instability strip centered around $11,000 \mathrm{~K}$, with most stars in it being variable. There is great interest in knowing if all the stars inside the instability strip are variable because, if so, the conclusions we derive from the DAVs are valid for all DAs. In this case the variability would be a normal stage of all DA stars as they move along the white dwarf cooling track. A second point is that a precise definition of the strip edges would help us in constraining convection theory for white dwarfs (Winget et al. 1982; Tassoul, Fontaine, \& Winget 1990). The ZZ Ceti stars have been used for measuring the evolutionary rate of these "lukewarm" white dwarfs (Kepler et al. 1991). The evolutionary time scale of white dwarfs have, in turn, been used as a tool to measure the age of the galactic disk (Winget et al. 1987; Iben \& Laughlin 1989; Yuan 1989; Wood 1992). Finally asteroseismology has been used on the pre-white dwarfs (PG 1159-035; Winget et al. 1991) and DBVs (GD358; Winget et al. 1992) to measure its structure and evolution.

The question of whether all stars inside the instability strip are variable will only be answered when we have good statistics on a large number of variables and nonvariables. To improve the statistics we have been obtaining time-series photometry of all DAs with colors close to the instability strip. In

\footnotetext{
1 CNPq Fellow.

${ }^{2}$ Visiting Astronomer at Laboratório Nacional de Astrofísica/Conselho Nacional de Desenvolvimento Científico-Brazil.

${ }^{3}$ Department of Astronomy, University of Texas at Austin, Austin, TX 78712.

${ }^{4}$ Visiting Astronomer, Cerro Tololo Inter-American Observatory, National Optical Astronomy Observatories, which are operated by the Association of Universities for Research in Astronomy, under contract with the National Science Foundation.
}

our work we have already found some nonpulsating stars in the instability strip (Kepler \& Nelan 1992), and in this paper we describe the discovery of the new DAV star, BPM 37093.

BPM 37093 is a southern hemisphere white dwarf identified as a proper motion star in the Bruce proper motion survey (Luyten 1941). Its coordinates are $\alpha_{1950}=12^{\mathrm{h}} 36^{\mathrm{m}} \cdot 1$ and $\delta_{1950}=-49^{\circ} 33^{\prime}$, and its magnitude is $V=13.96$ (Eggen 1969). Finding charts can be found in Luyten (1949) under the name L327-186. BPM 37093 is also cataloged as WD $1236-495$ by McCook \& Sion (1987, the white dwarf catalog). Even although BPM 37093 has been claimed as a nonvariable star (McGraw 1979), we decided to reobserve it to test for its variability for two reasons: (1) the report of McGraw claims it is nonvariable to a limit of $10 \mathrm{mmag}$. Since the discovery of G226-29 (McGraw \& Fontaine 1982) and the deciphering of its light curve (Kepler, Robinson, \& Nather 1983) we know that ZZ Ceti's can have amplitudes at least as low as 6 mmag; (2) in addition to being inside the strip in the Johnson limits determined by Fontaine et al. (1982): $0.16 \leq B-V \leq 0.25$, with $B-V=0.18$ (Eggen 1969); and in the Strömgren limits determined by Fontaine et al. (1985): $0.038 \leq b-y \leq 0.092$, with $b-y=0.070$ according to Wegner (1979), although $b-y=0.12$ according to Bessel \& Wickramasinghe (1978), BPM 37093 is also inside the ZZ Ceti instability strip according to the temperature determination by Kepler \& Nelan (1992) using IUE data. The authors determined temperatures in two ways: one using $I U E$ observations with the SWP image only, where they found the instability strip in the temperature range $11,560 \mathrm{~K} \leq T_{\text {eff }} \leq 13,150 \mathrm{~K}$ and the other using $\mathrm{SWP}$, LWP, and/or LWR, and Strömgren photometry; in this case the instability strip lies in the interval $10,300 \mathrm{~K} \leq T_{\text {eff }} \leq 13,150$ $\mathrm{K}$. The temperature determinations for BPM 37093 are 11,560 $\mathrm{K}$ (for the SWP fit only) and $11,200 \mathrm{~K}$ using the SWP + LWP + Strömgren data. In the first determination the star appears right at red edge of the instability strip, while in the second it appears well inside the instability strip.

\section{OBSERVATIONS AND ANALYSES}

During 1991 May we observed BPM 37093 using the $1.6 \mathrm{~m}$ telescope at LNA/CNPq (Laboratório Nacional de Astrofísica) in Brazil, equipped with a two-star photometer (Nather 1973) on two separate nights; one night we observed 
TABLE 1

JOURNAL OF OBSERVATIONS

\begin{tabular}{clcccc}
\hline \hline Run name & Observatory & $\begin{array}{c}\text { BJDD } \\
\text { at Start }\end{array}$ & $\begin{array}{c}\text { Length } \\
(\mathrm{s})\end{array}$ & $\begin{array}{c}\text { Integration } \\
\text { time (s) }\end{array}$ & Observers \\
\hline RA234 $\ldots \ldots \ldots$ & LNA $/ 1.6 \mathrm{~m}$ & 8395.546426 & 950 & 5 & AK, OG \\
RA237 ....... & LNA/1.6 m & 8396.446398 & 3625 & 5 & AK, OG \\
RA238 ....... & LNA/1.6 m & 8396.491072 & 3715 & 5 & AK, OG \\
RM101 ...... & CTIO $/ 1.0 \mathrm{~m}$ & 8469.494909 & 4990 & 10 & MD \\
RM102 ...... & CTIO $/ 1.0 \mathrm{~m}$ & 8469.465525 & 3410 & 5 & MD \\
\hline
\end{tabular}

the star for only 15 minutes (RA234), but we could already see its variation. During the next night we observed it (RA237 and RA238) for 2 hours. We did not get any other clear night during that run. For runs RA234, RA237, and RA238 we could see the variation even at the telescope and, of course, it appears clearly at the Fourier transform with no high-frequency peaks present.

In order to confirm our results, we reobserved BPM 37093 at CTIO with the $1.0 \mathrm{~m}$ telescope, using a single channel photometer. The data were taken with interruptions for sky readings and guiding. Due partially to telescope size and moonlight in the first run, the data from CTIO are noiser than that from LNA, but in both nights we detected again the same variation. A journal of all observations is presented in Table 1 .

We reduced our data with the standard procedure described in Kepler et al. (1982): sky subtraction by interpolation, and extinction correction by parabola fitting.

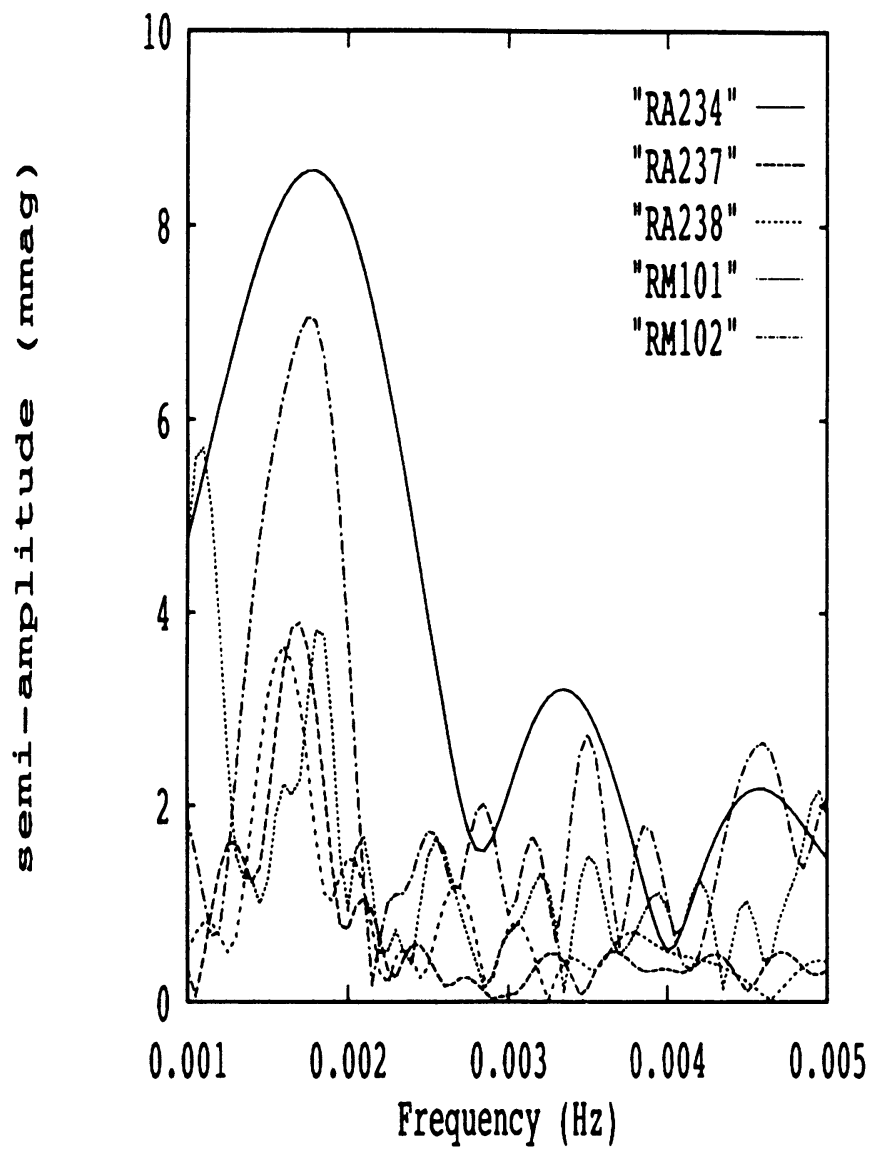

FIG. 1.-Fourier transforms of BPM 37093. Power spectra of the five runs shown separately.
We analyzed each light curve separately to verify the reproducibility of our results. In Figure 1 we show a portion of the Fourier transforms of the five light curves from Table 1; one can note the presence of the same peak with a period of $\sim 600 \mathrm{~s}$. The different amplitudes and frequencies of this peak are probably due to the presence of other periodicities, i.e., beating of two or more pulsations, and the varying width of the peak comes from differing lengths of the data. We infer the existence of more than one real frequency at this region from the Fourier transform of all our data set. In this Fourier transform the main peak at the region of $600 \mathrm{~s}$ is double; one peak has a semi-amplitude of $4.5 \mathrm{mmag}$ at $609 \mathrm{~s}$ and the other at $546 \mathrm{~s}$ with semi-amplitude of $\sim 4 \mathrm{mmag}$. On this transform we can see the peaks with a much better definition since the noise is not coherent over the whole data set. The peak at $609 \mathrm{~s}$ is now $20 \sigma$ over the noise, and the peak at $546 \mathrm{~s}$ is $10 \sigma$ above the noise. We show in Figure 2 a portion of the light curve of BPM 37093, during the best night we had.

\section{DISCUSSION}

We conclude that BPM 37093 is a real pulsator, with an amplitude of nearly $4 \mathrm{mmag}$ and a period around $600 \mathrm{~s}$. It is the $22 \mathrm{~d} \mathrm{ZZ}$ Ceti pulsator known. The low amplitude of its pulsations can be attributed to two causes: (1) an actually low amplitude pulsation on the surface of the star; and/or (2) its unfavorable geometrical orientation with respect to us; for example, if the angle between the line of sight and the rotational axis is near $90^{\circ}$, there is a geometrical cancellation of the brightness variations for modes symmetric in relation to the pole (see Pesnell 1985). This explanation is very unlikely, since we expect all different $m$ modes should also be excited and might then be observable.

The low amplitude of BPM 37093 pulsations encourages observers to reobserve some stars claimed as nonvariable which could be pulsating with an amplitude lower than the previous lower limit of 5 mmag used for most ZZ Ceti's searches. Another interesting point about the amplitude of the pulsations of BPM 37093 is that it seems contrary to the period-amplitude relation: the larger the periods, the larger the amplitudes (Winget \& Fontaine 1982). BPM 37093 has a long period and a very small amplitude. We suggest one way to understand this discrepancy: as a ZZ Ceti cools and advances through the red edge, their pulsations periods and amplitudes get larger, but there is a limit, the red edge. At the red edge damping starts to be more effective than driving, and the pulsations stop. What is not known is if the pulsations should disappear suddenly when the stars reach the red edge or should smoothly vanish. It is remarkable that, according to IUE SWP temperature, BPM 37093 defines the red edge of the instability strip. The discovery of a low-amplitude pulsator possibly located at the red edge adds some important clues to this 
No. 2, 1992

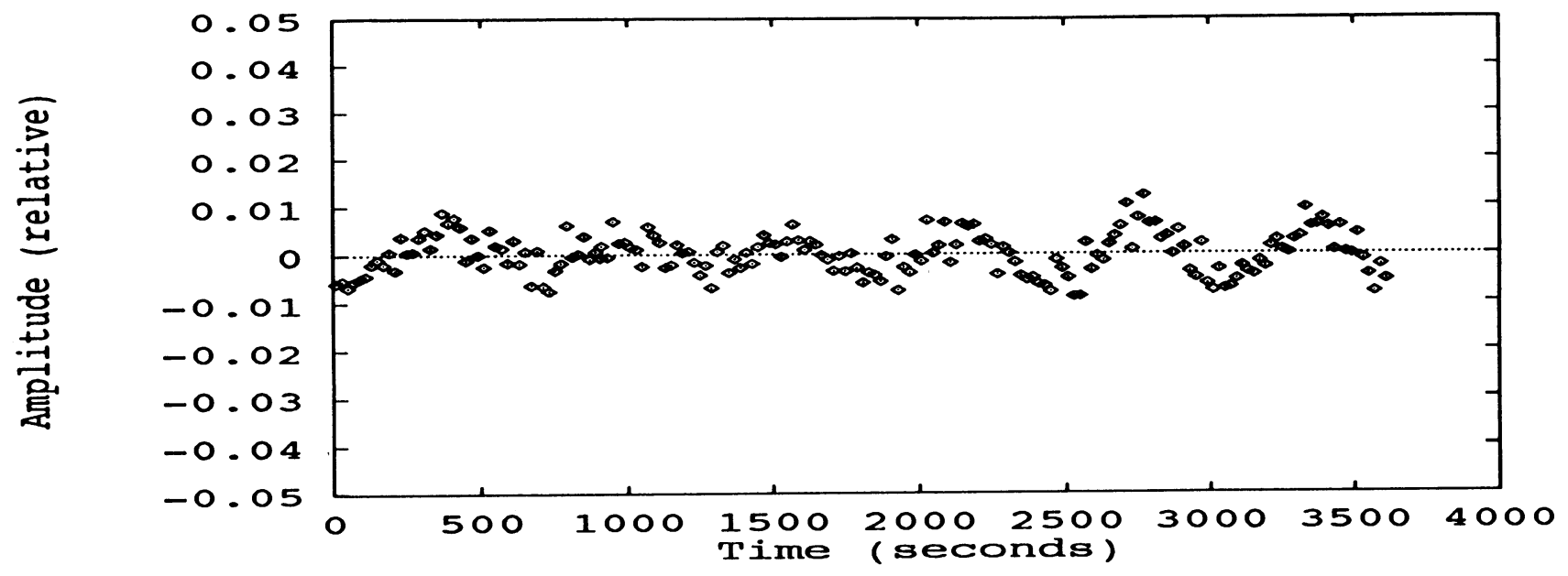

FIG. 2.-The light curve for run RA237, the best data taken on BPM 37093.

question; if BPM 37093 really is at the red edge, it indicates that the fate of pulsations in ZZ Ceti's is a slow process, rather than an abrupt one. Observations of this star in the coming years will tell us whether it has a low amplitude due to geometrical effects or to star related intrinsic characteristics. If the amplitude is really big, we then expect to see amplitude variations as in the other red edge large amplitude pulsators. If we do not observe these amplitude changes, then BPM 37093 is indeed an intrinsic small amplitude pulsator, ruling out the possibility of geometric effects. The confirmation if it really is at the very edge could be obtained from a study of uniform optical or IUE spectra of most ZZ Ceti stars.

This work was partially financed by $\mathrm{CNPq}$ (Brazil) and FINEP (Brazil). S. O. K. acknowledges the support of FCAR (Quebec-Canada) during his sabbatical at the Universite de Montréal, where the IUE studies were undertaken. We thank the director of CTIO for allocating us discretionary time at the $1 \mathrm{~m}$ telescope. We also thank G. Schumacher for modifying the data acquisition program at the CTIO $1 \mathrm{~m}$ for us.
Bessel, M. S., \& Wickramasinghe, D. T. 1978, MNRAS, 182, 275

Eggen, O. J. 1969, ApJ, 157, 287

Fontaine, G., Bergeron, P., Lacombe, P., Lamontagne, R., and Talon, A. 1985, AJ, 90, 1094.

Fontaine, G., McGraw, J. T., Dearborn, D. S. P., Gustafson, J., \& Lacombe, P. 1982, ApJ, 258, 651

Greenstein, J. L. 1982, ApJ, 258, 661

Iben, I., Jr., \& Laughlin, G. 1989, ApJ, 341, 312

Kepler, S. O., \& Nelan, E. P. 1992, AJ, submitted

Kepler, S. O., Robinson, E. L., \& Nather, R. E. 1983, ApJ, 271, 744

Kepler, S. O., Robinson, E. L., Nather, R. E., \& McGraw, J. T. 1982, ApJ, 254, 676

Kepler, S. O., et al. 1991, ApJ, 378, L45

Luyten, W. J. 1949, ApJ, 105, 528

Luyten, W. J. 1941, Bruce Proper Motion Survey, The General Catalogue

(Minneapolis: University of Minnesota Press)

McCook, G. P., \& Sion, E. M. 1987, ApJS, 65, 603

McGraw, J. T. 1979, ApJ, 229, 203

McGraw, J. T., \& Fontaine, G. 1982, private communication

\section{REFERENCES}

Nather, R. E. 1973, Vistas Astron., 15, 91

Pesnell, W. D. 1985, ApJ, 292, 238

Robinson, E. L. 1976, in Proc. Conf. on. Solar and Stellar Pulsations, ed N. Cox \& R. G. Deupree (Los Alamos Scientific Lab.)

Sion, E. M., Greenstein J. L., Landstreet, J. D., Liebert, J., Shipman, H. L., \& Wegner, G. 1983, ApJ, 269, 253

Tassoul, M., Fontaine, G., \& Winget, D. E. 1990, ApJS, 72, 335

Wegner, G. 1979, AJ, 84, 1384

Winget, D. E \& Fontaine, G. 1982, in Proc. Conf. on Pulsations in Classical and Cataclysmic Variables, ed. J. P. Cox \& C. J. Hansen (Boulder, CO: JILA), 46

Winget, D E., Hansen, C. J., Liebert, J., Van Horn, H. M., Fontaine, G., Nather, R. E. Kepler, S. O. \& Lamb, D. Q. 1987, ApJ, 315, L77

Winget, D. E. Van Horn, H. M., Tassoul, M., Hansen, C. J., Fontaine, G., \& Carrol, B. W. 1982, ApJ, 252, L65

Winget, D. E., et al. 1991, ApJ, 378, 326

. 1992, in preparation

Wood M A 1992, ApJ, 386, 539

Yuan, J. W. 1989, A\&A, 224, 108 